\title{
España y los 25 grandes de la ciencia mundial en cifras (1992-2008).
}

\author{
Por Emilio Delgado-López-Cózar, Evaristo Jiménez-Contreras y Rafael Ruiz-Pérez
}

\begin{abstract}
Resumen: Se presentan unas reflexiones a partir de la información suministrada por Thomson Reuters de manera gratuita en ScienceWatch.com. Se muestran los rankings de producción y citación de los 24 países con mayor actividad científica del mundo. En este contexto se analiza con especial detalle la evolución seguida por España.

Palabras clave: Bibliometría, Análisis de citas, Publicación científica, Evaluación del rendimiento investigador, Thomson Scientific, ScienceWatch.com, Essential science indicators, España.
\end{abstract}

Title: Spain and the world's 25 top science countries in figures (1992-2008)

Abstract: Production and citation rankings of the top 25 world science countries are presented from the free data provided by Thomson Reuters in ScienceWatch.com. The development of Spain is discussed in detail.

Keywords: Bibliometrics, Citation analysis, Scientific publication, Scientific performance, Thomson Reuters, ScienceWatch.com, Essential science indicators, Spain.

Delgado-López-Cózar, Emilio; Jiménez-Contreras, Evaristo; Ruiz-Pérez, Rafael. "España y los 25 grandes de la ciencia mundial en cifras (1992-2008)". El profesional de la información, 2009, enero-febrero, v. 18, n. 1, pp. 81-86.

DOI: 10.3145/epi.2009.ene.11

TODAVÍA TENEMOS IMPRESIONADA NUESTRA RETINA con el medallero de los Juegos Olímpicos de Pekín -el podium donde se dirimen los honores patrios- $y$ el subsiguiente debate sobre el ascenso de China a la condición de primera potencia deportiva del mundo desbancando a Estados Unidos, con el estancamiento de España (18 medallas, puesto 14).

Y mientras continuamos impresionados con la tormenta financiera y sus repercusiones en la economía mundial, hemos asistido recientemente a una nueva polémica que ha acaparado el debate político español durante varias semanas: ¿Por qué España no estuvo inicialmente invitada a la Cumbre de Washington? ¿Por qué no figuraba en el G20, siendo la octava economía del mundo por PIB? ¿Cuál es la potencial real de España en el concierto de las naciones? De pronto el ciudadano de a pie ha descubierto que España no está en ningún $\mathrm{G}$ conocido.
Por lo tanto, hablar sobre la posición que ocupa España, en este caso en el mundo de la ciencia, es un tema de viva actualidad.

El hábito anglosajón de clasificar competitivamente todo lo mensurable se ha convertido en el medio habitual para determinar el poderío de las naciones en todos los órdenes de la vida: economía, sociedad, cultura, ocio, ... La ciencia no es una excepción y participa en estos juegos con entusiasmo (May, 1997; King, 2004).

Las publicaciones científicas, en la medida en que constituyen el principal canal para difundir y validar los resultados de la investigación, conforman probablemente de entre los distintos resultados a los que da lugar la actividad investigadora (patentes, nuevos productos, métodos...), el indicador clave para medir la capacidad de generar conocimiento que posee cualquier actor científico (autor, institución o país). Por eso el recuento de publicacio- nes, especialmente el de artículos científicos y las citas a las que éstos dan lugar se han convertido en indicadores bibliométricos cruciales para determinar la productividad, visibilidad, impacto e influencia de la actividad científica, sin olvidar la facilidad con que se pueden procesar y su objetividad, más aparente que real pero no caprichosa.

"En bibliometría hay dos medidas básicas: contar el número de publicaciones producidas y el de citas recibidas"

\section{WoS, base de datos clave}

Web of Science es la fuente de información indispensable para realizar estos recuentos. Tanto la Ocde, el organismo internacional de referencia en estos temas, la Unión 
Europea a través de sus European reports on science \& technology indicators, como Estados Unidos con sus Science and engineering indicators producidos por la National Science Foundation, o el Reino Unido a través de los Research assessment exercises (RAE) o Japón con sus Science and technology indicators generados por el National Institute of Science and Technology Policy in Japan, vienen empleando WoS como fuente para obtener sus indicadores. Lo mismo ocurre con los principales autores y grupos de investigación bibliométrica en el mundo (Henk Moed, Anthony F. J. Van Raan, Wolfgang Glänzel, Loet Leydesdorff, Andras Schubert...). Y por último conviene señalar que los dos rankings más reputados de universidades se basan en información suministrada por WoS: tanto el elaborado por el Centre for Science and Technology Studies (CWTS) de la Leiden University, como el de la Jiao Tong University in Shanghai.

http://www.oecd.org/topic/0,3373,en_ 2649_37417_1_1_1_1_37417,00. html

http://cordis.europa.eu/indicators/ publications.htm

http://www.nsf.gov/statistics/seind08/ c5/c5h.htm

http://www.rae.ac.uk/

http://www.nistep.go.jp/index-e.html http://www.socialsciences.leidenuniv. $n l$

\section{http://www.arwu.org}

España, que por fortuna no es diferente en este caso, utiliza también WoS como base de los indicadores bibliométricos elaborados por el Micinn (Ministerio de Ciencia e Innovación), el INE (Instituto Nacional de Estadística), Cotec (Fundación para la innovación tecnológica), el Sistema Integral de Seguimiento y Evaluación (Sise), el Csic o el Instituto de estudios documentales sobre ciencia y tecnología (Iedcyt).

http://www.micinn.es/ciencia/ estadisticas/files/2008-indicadores-
sistema-Espanol-ciencia-tecnologia07.pdf

http://www.cotec.es

http://sise.fecyt.es

http://www.cindoc.csic.es

\section{ScienceWatch.com}

Como ya se señaló en un artículo anterior (Ruiz et al, 2008), Thomson Reuters (TR) ofrece información bibliométrica gratuita a través del portal ScienceWatch.com. Desde hace ya siete años (2002) en diciembre presenta la lista de los 20 países punteros en artículos y citas que hayan publicado al menos 10.000 trabajos en los 10 últimos años. Son datos extraídos de los Essential science indicators (ESI), producto de pago incluido dentro de la licencia del Web of Knowledge de TR, donde figuran los 147 países más citados del planeta.

En diciembre de 2008 se publicaron los datos referidos al período 1998-2008. Con esta información y con la aparecida desde 2002 hemos confeccionado varias tablas que nos permitirán conocer no sólo la posición de España, sino la del resto de los países desarrollados, así como los cambios operados en la publicación científica en el mundo.

http://sciencewatch.com/dr/cou/2008/ O8decALL

http://www.in-cites.com/countries/ 2007allfields.html

En dicho informe los países se encuentran ordenados de acuerdo con tres medidas: el número total de trabajos (tabla 1), el número total de citas (tabla 2) y el promedio de citas por trabajo (tabla 3 ).

\section{Artículos y sus citas}

En bibliometría las medidas más elementales son dos: el número de publicaciones producidas y el de citas recibidas. Mientras que el primero es un indicador estimativo del grado de actividad y del volumen del conocimiento producido, el segundo lo es de la cantidad de conocimiento transferido y utilizado. Los dos indicadores nos hablan de volumen porque responden a una ecuación que es básica a la hora de medir la ciencia: mayores gastos e inversiones en $\mathrm{I}+\mathrm{D}+\mathrm{I}$, determinan un mayor número de científicos; un mayor número de científicos supone un mayor número de publicaciones; y éstas a su vez un mayor número de citas. En resumidas cuentas, tanto el número de publicaciones como el de citas son una función del tamaño de las comunidades científicas, que a su vez viene determinado por el total de inversiones en ciencia, que a su vez es el reflejo de la riqueza de un país. Por lo tanto estos dos indicadores cuantifican lo poderosas e influyentes que son las respectivas comunidades científicas.

Tomados así, como indicadores del volumen de la actividad científica, las tablas 1 y 2 nos muestran los 25 países más avanzados del mundo. Sería el G25 de la ciencia, por utilizar esa terminología al uso. De la lectura de estas tablas se desprenden los siguientes hechos:

- España se sitúa en la década 1998-2008 en el $9^{\circ}$ puesto del mundo y en el $5^{\circ}$ de la Unión Europea por producción, y en el $11^{\circ}$ y $6^{\circ}$ respectivamente, por citación. Avanza en el ranking mundial dos puestos en producción y uno en citación desde la década 1992-2002. Lo más reseñable es que es el quinto país que más crece, y el primer europeo, y comparte con Polonia unas tasas de crecimiento que casi triplican en producción y duplican en citación a las de los más poderosos del mundo.

- Estados Unidos sigue siendo quien tiene mayor volumen de

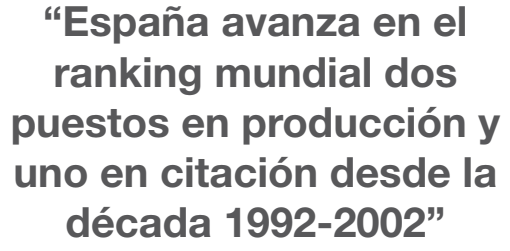

"España avanza en el ranking mundial dos puestos en producción y uno en citación desde la década 1992-2002" 
producción y citación científica. Por sí solo representa un tercio de la producción y un $40 \%$ de la citación científica mundial. Ahora bien, es el país que menos crece y marca un claro retroceso en su peso en la ciencia mundial: si en la década de 1992-2002 representaba el 34\% y $44 \%$ de la producción y citación mundial, en el decenio 1998-2008 cae al $30 \%$ y $38 \%$.

- El hecho más destacado es la irrupción del frente asiático en los puestos de cabeza de la ciencia en el mundo con China, Corea del Sur, India y Taiwán como abanderadas. Si en la década 1992-2002 estos países representaban el $7 \%$ de la producción y el $2 \%$ de la citación mundial, en 1998-2008 ya suponen el 12\% y el 5,3\% respectivamente. Los crecimientos de Corea del Sur y, sobre todo, de China son espectaculares pues han multiplicado por tres su producción y por cinco su citación. Con estos resultados China ha escalado del puesto $10^{\circ}$ al $5^{\circ}$ del mundo en producción y del $20^{\circ}$ al $10^{\circ}$ en citación. Si nos refiriéramos sólo al año 2008, China ya es la segunda potencia del mundo en producción tras Estados Unidos. También es digno de mención el crecimiento de países emergentes como Brasil. Y

\section{"Los crecimientos de Corea del Sur y China son espectaculares pues han multiplicado por tres su producción y por cinco su citación"}

llamamos la atención sobre Turquía, que muy pronto figurará en esta lista. De hecho en 2008 ya está entre los 20 países más productivos.

- Por último, constatar que todos los países crecen. La explicación es doble: más artículos en la base de datos pero, sobre todo más autores y por lo tanto instituciones y países por artículo. Según el recuento total (a cada país participante en un trabajo se le atribuye todo el crédito, independientemente de su grado de participación) lo que crecen son las colaboraciones en los artículos más que los artículos mismos. En definitiva más comensales para el mismo pastel. No obstante conviene advertir que no todos los países crecen de la misma forma. Unos lo hacen más que otros: China, Corea del Sur, Brasil, Taiwán, y

\begin{tabular}{|c|c|c|c|c|c|c|c|c|}
\hline Países & $\begin{array}{c}1992 \\
- \\
2002\end{array}$ & $\begin{array}{c}1993 \\
- \\
2003\end{array}$ & $\begin{array}{c}1994 \\
- \\
2004\end{array}$ & $\begin{array}{l}1995 \\
- \\
2005\end{array}$ & $\begin{array}{l}1996 \\
- \\
2006\end{array}$ & $\begin{array}{c}1997 \\
- \\
2007\end{array}$ & $\begin{array}{c}1998 \\
- \\
2008\end{array}$ & $\begin{array}{c}\text { Tasa de crecimiento } \\
1992 / 02-1998 / 08 \\
\text { en } \%\end{array}$ \\
\hline Estados Unidos & 2.618 .154 & 2.705 .352 & 2.698 .434 & 2.803 .625 & 2.831 .004 & 2.864 .275 & 2.959 .661 & 13,0 \\
\hline Japón & 672.308 & 713.542 & 722.512 & 759.989 & 771.573 & 777.992 & 796.807 & 18,5 \\
\hline Alemania & 619.323 & 655.586 & 666.104 & 705.953 & 723.435 & 738.067 & 766.146 & 23,7 \\
\hline Reino Unido & 570.667 & 598.470 & 604.397 & 633.123 & 643.557 & 653.177 & 678.686 & 18,9 \\
\hline China & 193.691 & 236.996 & 271.032 & 340.206 & 400.917 & 471.890 & 573.486 & 196,1 \\
\hline Francia & 459.963 & 484.291 & 488.585 & 513.387 & 522.015 & 529.636 & 548.279 & 19,2 \\
\hline Canadá & 346.126 & 358.007 & 358.176 & 375.239 & 383.199 & 393.143 & 414.248 & 19,7 \\
\hline Italia & 288.763 & 310.557 & 320.667 & 344.759 & 358.452 & 371.205 & 394.428 & 36,6 \\
\hline España & 191.422 & 209.762 & 219.404 & 240.618 & 254.808 & 270.139 & 292.146 & 52,6 \\
\hline Rusia & 255.548 & 285.856 & 282.027 & 286.481 & 280.480 & 275.945 & 276.801 & 8,3 \\
\hline Australia & 198.006 & 211.549 & 216.819 & 232.423 & 240.738 & 249.892 & 267.134 & 34,9 \\
\hline India & 168.561 & 177.687 & 180.783 & 194.593 & 203.989 & 215.847 & 237.364 & 40,8 \\
\hline Holanda & 184.526 & 194.710 & 197.426 & 209.233 & 215.050 & 220.881 & 231.682 & 25,6 \\
\hline Corea del Sur & 90.907 & 111.406 & 126.438 & 153.051 & 173.050 & 192.361 & 218.077 & 139,9 \\
\hline Suecia & 144.425 & 152.632 & 154.372 & 162.696 & 165.862 & 168.574 & 174.418 & 20,8 \\
\hline Suiza & 129.785 & 137.661 & 140.164 & 149.375 & 154.291 & 159.667 & 168.527 & 29,9 \\
\hline Brasil & & & 98.747 & 114.192 & 125.132 & 137.159 & 157.860 & $* 59,9$ \\
\hline Taiwán & & & 98.314 & 110.948 & 120.447 & 130.281 & 144.807 & $* 47,2$ \\
\hline Polonia & 85.445 & 94.101 & 98.685 & 109.690 & 115.535 & 121.061 & 131.646 & 54,1 \\
\hline Bélgica & 92.266 & 99.226 & 102.169 & 110.151 & 114.172 & 118.411 & 125.520 & 36,0 \\
\hline Israel & 90.514 & 95.942 & 96.890 & 102.364 & 104.058 & 106.122 & 109.637 & 21,1 \\
\hline Escocia & 88.836 & 93.327 & 94.199 & 98.816 & 100.526 & 102.053 & 106.209 & 19,6 \\
\hline Dinamarca & 72.630 & 76.889 & 78.416 & 82.950 & 85.234 & 87.496 & 91.670 & 26,2 \\
\hline Austria & 63.319 & 68.610 & 71.227 & 77.050 & & 83.179 & 87.953 & 38,9 \\
\hline Finlandia & 66.524 & 71.328 & 73.068 & 77.650 & 79.788 & 82.001 & 85.567 & 28,6 \\
\hline
\end{tabular}

Tabla 1. Los 25 países con más producción científica en los Essential science indicators ordenados según número de trabajos.

*Tasa de crecimiento del periodo 1998-2008 con relación a 1994-2004 
lo trascendente para nosotros es que España está entre ellos. En cambio, los países que menos crecen son las grandes potencias científicas (Japón, Inglaterra, Francia, Canadá) con Estados Unidos a la cabeza. Y es que cuando se está arriba es más difícil crecer, se está prácticamente en el nivel de saturación del que hablaba Price.

\section{Citas por artículo}

El número de citas, por ser una medición bruta, es un indicador bastante tosco que conviene pulir. Y para ello nada mejor que relativizarlo por el número de trabajos. De esta forma podemos decir de un país cuál es el número de citas promedio que obtienen los artículos que produce. Es un indicador que cualifica además de cuantificar. Y aquí sí que podemos hablar ya sin ambages de visibilidad e impacto. Es evidente que cuando un científico cita un trabajo anterior lo que formalmente hace es reconocer la influencia que dicho trabajo ha tenido en el suyo propio. Si se acepta esta premisa, los trabajos más citados tienen una influencia mayor en las actividades investigadoras que los que tienen menos citas. De ahí que un mayor impacto promedio de los artículos de un país sea indicativo de una mayor influencia de este país como potencia científica.

En la tabla 3, en la que se ordenan los países por el promedio de citas que tienen sus trabajos, nos encontramos con un panorama sensiblemente diferente al descrito anteriormente. Y decimos sensiblemente porque lo que ocurre es que las grandes potencias científicas (Alemania, Japón, Francia, Inglaterra, Canadá) - a excepción de Estados Unidos- bajan bastante en el escalafón y dejan su sitio a las pequeñas potencias científicas (Suiza, Holanda y los países escandinavos) que escalan a las primeras posiciones. Por el contrario, los países emergentes, con los asiáticos a la cabeza, se van a la cola de la lista. Aparte de este fenómeno general se detectan otros hechos interesantes:

- Ordenados los países según este indicador de citas/trabajo, España cae al puesto $18^{\circ}$ del mundo en la década 1998-2008, perdiendo nueve puestos respecto del ranking de producción mundial. Le sucede algo parecido en el conjunto europeo, retrocediendo hasta el puesto $10^{\circ}$ de la Unión Europea. Debemos

\begin{tabular}{|c|c|c|c|c|c|c|c|c|}
\hline Países & $\begin{array}{c}1992 \\
- \\
2002\end{array}$ & $\begin{array}{c}1994 \\
- \\
2004\end{array}$ & $\begin{array}{c}1993 \\
- \\
2003\end{array}$ & $\begin{array}{c}1995 \\
- \\
2005\end{array}$ & $\begin{array}{c}1996 \\
- \\
2006\end{array}$ & $\begin{array}{c}1997 \\
- \\
2007\end{array}$ & $\begin{array}{c}1998 \\
- \\
2008\end{array}$ & $\begin{array}{c}\text { Tasa de crecimiento } \\
1992 / 02-1998 / 08 \\
\text { en } \%\end{array}$ \\
\hline Estados Unidos & 30.765 .049 & 33.212 .308 & 33.089 .756 & 36.231 .437 & 37.822 .213 & 39.027 .838 & 42.269 .694 & 37,4 \\
\hline Alemania & 5.186 .228 & 6.102 .642 & 5.857 .244 & 6.928 .683 & 7.497 .007 & 7.935 .567 & 8.787 .460 & 69,4 \\
\hline Inglaterra & 5.628 .105 & 6.373 .300 & 6.212 .840 & 7.097.782 & 7.565 .163 & 7.955 .521 & 8.768 .475 & 55,8 \\
\hline Japón & 4.591 .831 & 5.264 .781 & 5.098 .499 & 5.898 .079 & 6.298 .466 & 6.612 .826 & 7.201 .664 & 56,8 \\
\hline Francia & 3.777 .753 & 4.338 .642 & 4.213 .581 & 4.847 .515 & 5.171 .849 & 5.414 .557 & 5.933 .187 & 57,1 \\
\hline Canadá & 3.259 .935 & 3.587 .966 & 3.549 .116 & 3.958 .929 & 4.194 .095 & 4.377 .986 & 4.837 .825 & 48,6 \\
\hline Italia & 2.245 .050 & 2.709.842 & 2.569 .970 & 3.084 .580 & 3.363 .216 & 3.594 .444 & 4.044 .512 & 80,1 \\
\hline Holanda & 1.908 .540 & 2.206 .097 & 2.135 .032 & 2.484 .821 & 2.664 .587 & 2.837 .971 & 3.148 .005 & 65,0 \\
\hline Australia & 1.523 .844 & 1.821 .757 & 1.736 .998 & 2.086 .047 & 2.272 .955 & 2.442 .466 & 2.784 .738 & 82,7 \\
\hline China & 494.157 & 799.415 & 658.355 & 1.129 .014 & 1.480 .743 & 1.894 .810 & 2.646 .085 & 435,6 \\
\hline España & 1.200 .295 & 1.529 .708 & 1.419 .447 & 1.810 .352 & 2.041 .705 & 2.248 .541 & 2.602 .330 & 116,8 \\
\hline Suiza & 1.585 .691 & 1.823 .353 & 1.769 .220 & 2.028 .778 & 2.168 .127 & 2.285 .847 & 2.502 .210 & 57,8 \\
\hline Suecia & 1.446 .651 & 1.645 .518 & 1.600 .307 & 1.841 .611 & 1.960 .099 & 2.053 .237 & 2.257 .641 & 56,1 \\
\hline Bélgica & 825.768 & 982.002 & 935.873 & 1.112 .803 & 1.206 .208 & 1.295 .296 & 1.461 .478 & 77,1 \\
\hline Escocia & 873.438 & 1.001 .777 & 967.215 & 1.129 .560 & 1.212 .942 & 1.286 .716 & 1.422 .252 & 62,9 \\
\hline Dinamarca & 735.002 & 856.210 & 827.292 & 972.211 & 1.052 .389 & 1.129 .465 & 1.262 .693 & 71,7 \\
\hline Corea del Sur & 308.063 & 504.634 & 420.349 & 678.175 & 840.487 & 1.005 .008 & 1.256 .724 & 307,8 \\
\hline Israel & 749.527 & 864.214 & 835.818 & 978.865 & 1.039 .021 & 1.098 .417 & 1.210 .807 & 61,5 \\
\hline Rusia & 665.442 & 870.485 & 848.345 & 971.561 & 1.019 .009 & 1.057 .928 & 1.135 .496 & 70,6 \\
\hline India & 471.413 & 573.792 & 538.739 & 687.523 & 788.852 & 895.528 & 1.088 .425 & 131,0 \\
\hline Finlandia & 610.841 & 733.391 & 700.902 & 831.065 & 892.853 & 948.501 & 1.038 .721 & 70,2 \\
\hline Austria & 499.319 & 574.298 & 611.484 & 716.966 & & 859.140 & 974.554 & 95,2 \\
\hline Brasil & & 433.772 & & 540.161 & 627.441 & 720.131 & 880.821 & $* 102,1$ \\
\hline Taiwán & & 430.290 & & 524.438 & 608.561 & 693.017 & 828.751 & $* 92,6$ \\
\hline Polonia & 359.420 & 453.940 & 419.948 & 537.479 & 597.312 & 658.927 & 766.033 & 113,3 \\
\hline
\end{tabular}

Tabla 2. Los 25 países más citados en los Essential science indicators ordenados según número de citas.

*Tasa de crecimiento relativa al periodo 1994-2004 a 1998-2008 
advertir que ésta es su posición de entre los 25 países con más actividad científica del mundo, porque su puesto real cuando se contabilizan otros países occidentales como puede ser Noruega, es muy inferior, cayendo por debajo del puesto 25 . Por otra parte, su posición no ha variado en estos años. Sí podemos decir que ha acortado la distancia respecto al país que le precede, que es Japón. Esto se debe a que sigue estando entre los cinco países que más crecen en estos años, con unas tasas de incremento que casi duplican a las de los países más poderosos del mundo.

- Estados Unidos, a pesar de que no es el país puntero en esta medida por apenas unas décimas, es indudablemente el más influyente del planeta. Es admirable que

\section{"En el indicador de citas/ trabajo, España cae al puesto $18^{\circ}$ del mundo, perdiendo nueve respecto del ranking de producción mundial"}

teniendo cuatro veces más producción que Japón, que es el país que le sigue en volumen y reuniendo por sí mismo la misma producción que Japón, Alemania, Inglaterra y Francia juntos, sea capaz de mantener una citación por trabajo tan elevada. Hay que destacar el descenso de países como Japón, que bajan estrepitosamente en este indicador, o el de Inglaterra, Alemania o Francia. Por lo tanto, a pesar de los descensos brutos en producción y citación, la fortaleza de Estados Unidos como potencia científica sigue intacta.

- Si el crecimiento de China, Corea del Sur, India y Taiwán en producción y citación es importante, no se produce con los mismos ritmos en las citas por trabajo. Sin duda también en este indicador crecen más que nadie, y como consecuencia de ello están reduciendo el diferencial que les separa con los países occidentales. Y es que el impacto y la influencia real en la ciencia no se improvisan, es algo de crecimiento lento, requiere un tiempo largo como diría Fernand Braudel. Como demostración de lo que decimos, observamos que no hay variaciones en el escalafón ni para los países de la cabeza ni de la cola.

\begin{tabular}{|c|c|c|c|c|c|c|c|c|}
\hline Países & $\begin{array}{c}1992 \\
- \\
2002\end{array}$ & $\begin{array}{c}1993 \\
- \\
2003\end{array}$ & $\begin{array}{c}1994 \\
- \\
2004\end{array}$ & $\begin{array}{c}1995 \\
- \\
2005\end{array}$ & $\begin{array}{l}1996 \\
- \\
2006\end{array}$ & $\begin{array}{c}1997 \\
- \\
2007\end{array}$ & $\begin{array}{c}1998 \\
- \\
2008\end{array}$ & $\begin{array}{c}\text { Tasa de crecimiento } \\
1992 / 02-1998 / 08 \\
\text { en } \%\end{array}$ \\
\hline Suiza & 12,22 & 12,85 & 13,01 & 13,58 & 14,05 & 14,32 & 14,85 & 21,5 \\
\hline Estados Unidos & 11,75 & 12,23 & 12,31 & 12,92 & 13,36 & 13,63 & 14,28 & 21,5 \\
\hline Dinamarca & 10,12 & 10,76 & 10,92 & 11,72 & 12,35 & 12,91 & 13,77 & 27,1 \\
\hline Holanda & 10,34 & 10,97 & 11,17 & 11,88 & 12,39 & 12,85 & 13,59 & 31,4 \\
\hline Escocia & 9,83 & 10,36 & 10,63 & 11,43 & 12,07 & 12,61 & 13,39 & 36,2 \\
\hline Suecia & 10,02 & 10,48 & 10,66 & 11,32 & 11,82 & 12,18 & 12,94 & 29,1 \\
\hline Inglaterra & 9,86 & 10,38 & 10,54 & 11,21 & 11,76 & 12,18 & 12,92 & 31,0 \\
\hline Finlandia & 9,18 & 9,83 & 10,04 & 10,70 & 11,19 & 11,57 & 12,14 & 32,2 \\
\hline Canadá & 9,42 & 9,91 & 10,02 & 10,55 & 10,94 & 11,14 & 11,68 & 24,0 \\
\hline Bélgica & 8,95 & 9,43 & 9,61 & 10,10 & 10,56 & 10,94 & 11,64 & 30,1 \\
\hline Alemania & 8,37 & 8,93 & 9,16 & 9,81 & 10,36 & 10,75 & 11,47 & 37,0 \\
\hline Austria & 7,89 & 8,37 & 8,59 & 9,31 & & 10,33 & 11,08 & 29,0 \\
\hline Israel & 8,28 & 8,71 & 8,92 & 9,56 & 9,99 & 10,35 & 11,04 & 33,3 \\
\hline Francia & 8,21 & 8,70 & 8,88 & 9,44 & 9,91 & 10,22 & 10,82 & 31,8 \\
\hline Australia & 7,70 & 8,21 & 8,40 & 8,98 & 9,44 & 9,77 & 10,42 & 35,3 \\
\hline Italia & 7,77 & 8,28 & 8,45 & 8,95 & 9,38 & 9,68 & 10,25 & 31,9 \\
\hline Japón & 6,83 & 7,15 & 7,29 & 7,76 & 8,16 & 8,50 & 9,04 & 32,4 \\
\hline España & 6,27 & 6,77 & 6,97 & 7,52 & 8,01 & 8,32 & 8,91 & 42,1 \\
\hline Polonia & 4,21 & 4,46 & 4,60 & 4,90 & 5,17 & 5,44 & 5,82 & 38,2 \\
\hline Corea del Sur & 3,39 & 3,77 & 3,99 & 4,43 & 4,86 & 5,22 & 5,76 & 69,9 \\
\hline Taiwán & & & 4,38 & 4,73 & 5,05 & 5,32 & 5,72 & $* 30,6$ \\
\hline Brasil & & & 4,39 & 4,73 & 5,01 & 5,25 & 5,58 & $* 27,1$ \\
\hline China & 2,55 & 2,78 & 2,95 & 3,32 & 3,69 & 4,02 & 4,61 & 80,8 \\
\hline India & 2,80 & 3,03 & 3,17 & 3,53 & 3,87 & 4,15 & 4,59 & 63,9 \\
\hline Rusia & 2,60 & 2,97 & 3,09 & 3,39 & 3,63 & 3,83 & 4,10 & 57,7 \\
\hline
\end{tabular}

Tabla 3. Los 25 países más citados en los Essential science indicators ordenados según el promedio de citas por trabajo.

*Tasa de crecimiento del periodo 1998-2008 en relación con 1994-2004 


\section{"La fortaleza de Estados Unidos como potencia científica sigue intacta"}

- Y por último, constatar de nuevo cómo el crecimiento en este indicador es general: todos los países crecen. Pero evidentemente no lo hacen de la misma manera. Los de abajo (China, Corea del Sur, Rusia, India y España) crecen mucho más que los de arriba (Suiza, Estados Unidos, Dinamarca, Suecia). Y es que, como ya decíamos antes, cuando se está arriba es más difícil crecer. En definitiva se está produciendo una convergencia general de forma que la citación promedio de los tres últimos países (China, India y Rusia) respecto de los tres primeros (Suiza, Estados Unidos y Dinamarca) ha pasado de representar el $23 \%$ en la década 1992-2002 al 30\% en la década 1998-2008.

\section{Consideraciones finales}

No quisiéramos terminar este breve ensayo sin recordar unas premisas a la luz de las cuales debe leerse cualquier tipo de ranking bibliométrico como los que aquí hemos presentado.

Hay que tener siempre presente que los indicadores bibliométricos son estimadores parciales de la generación y difusión del conocimiento y que deberían ser combinados con otros tipos de aproximaciones y datos.

En bibliometría nunca se debe olvidar que los resultados son directamente proporcionales a la cobertura y calidad de la fuente de donde se extraen los datos (Moed et al, 1995). A pesar de ser $W o S$ el sistema que indiza la mejor literatura científica del mundo, hay que recordar que en ella figura sólo una parte del conocimiento registrado y codificado: el que se publica en revistas, fundamentalmente de ámbito internacional. Esta base presenta un sesgo anglosajón, un predominio de la ciencia básica sobre la aplicada, e igualmente ofrece una mayor representación de las ciencias naturales frente a las sociales y humanidades. Aun con estas limitaciones, $W o S$ es un insuperado escenario de análisis, la referencia aceptada en el ámbito internacional para el seguimiento de la actividad científica y, en cualquier caso, en ella están muy bien representados los ejes de la ciencia que hacen girar la rueda del progreso científico y tecnológico.

Por otra parte, aunque pueda resultar reiterativo, conviene recalcar que se han presentado datos brutos y que el sistema de recuento empleado es el total, de modo que cuando un trabajo es firmado por varios autores pertenecientes a distintas instituciones y países se atribuye a cada uno de éstos el trabajo completo. No se atiende por tanto al grado de participación o responsabilidad en el mismo fraccionando la asignación en función del número de participantes. Un recuento fraccionado o de otro tipo podría producir resultados algo distintos, aunque no sustancialmente.

La científica es una actividad económica y social más, y por ello hay que correlacionarla con los principales indicadores socioeconómicos. Tradicionalmente se viene relativizando con tres variables: población total, gasto total en I+D+I y, sobre todo, personal dedicado a la investigación con dedicación a tiempo completo. Cuando se relativiza la producción científica con estos indicadores se obtiene una visión más objetiva, y seguramente más útil de la productividad (qué resultados con qué recursos) y eficiencia (obtener más con menos) de un país.

Queda para otro trabajo la realización de estas ponderaciones. En éste realmente sólo pretendíamos mostrar cómo a partir de la información ofrecida gratuitamente por $T R$ en ScienceWatch.com se puede retratar sin más coste que un mero análisis contable la evolución de la actividad científica española y la del mundo.

\section{Bibliografía}

European Commission. Directorate General for Research. Key Figures 2007: Towards a European Research Area Science, Technology and Innovation. Luxembourg: Office for Official Publications of the European Communities, 2007. ISBN 92-79-03450-2. ftp://ftp.cordis.europa. eu/pub/era/docs/keyfigures_2007.pdf

King, D. A. "The scientific impact of nations". Nature, 2004, v. 430, pp. 311-316.

May, R. "The scientific wealth of nations". Science. 1997, v. 275, n. 5301, pp. 793-796.

Moed, H.; De Bruin, R.; Van Leeuwen, Th. N. "New bibliometric tools for the assessment of national research performance: database description, overview of indicators and first applications". Scientometrics, 1995, v. 33, n. 3, pp. 381-422.

Ruiz-Pérez, R.; Jiménez-Contreras, E.; Delgado-López-Cózar, E. "Complementos bibliométricos de Thomson Scientific en la web: buenos, bonitos y gratuitos". El profesional de la información, 2008, v. 17, n. 5, pp. 553-557.

Wikipedia, Fernand Braudel

http://es.wikipedia.org/wiki/Fernand_Braudel

Fuente de datos: ScienceWatch.com:

http://www.in-cites.com/countries/2007allfields. html

http://www.in-cites.com/countries/2006allfields. html

http://www.in-cites.com/countries/2005allfields. html

http://www.in-cites.com/countries/2004allfields. html

http://www.in-cites.com/countries/2003allfields. html

http://www.in-cites.com/countries/2002allfields. html

EmilioDelgado-López-Cózar, Evaristo Jiménez-Contreras, Rafael Ruiz-Pérez

EC3: Evaluación de la Ciencia y de la Comunicación Científica, Departamento de biblioteconomía y documentación, Universidad de Granada, Campus Cartuja.

18071 Granada

edelgado@ugr.es

evaristo@ugr.es

rruiz@ugr.es 